\title{
Antifungal Treatment With Carvacrol and Eugenol of Oral Candidiasis in Immunosuppressed Rats
}

N. Chami ${ }^{1}$, F. Chami ${ }^{1}$, S. Bennis ${ }^{1}$, J. Trouillas ${ }^{2}$ and A. Remmal ${ }^{1}$

\author{
${ }^{1}$ Faculté des Sciences Fès Laboratoire de Biotechnologie BP \\ 1796 Atlas FES, Morocco; ${ }^{2}$ Faculté de Médecine Lyon--RTH \\ Laennec Laboratoire d'Histologie Embryologie Moléculaires, \\ Lyon, France
}

\begin{abstract}
Carvacrol and eugenol, the main (phenolic) components of essential oils of some aromatic plants, were evaluated for their therapeutic efficacy in the treatment of experimental oral candidiasis induced by Candida albicans in immunosuppressed rats. This anticandidal activity was analyzed by microbiological and histopathological techniques, and it was compared with that of nystatin, which was used as a positive control. Microbiologically, carvacrol and eugenol significantly $(p<0.05)$ reduced the number of colony forming units (CFU) sampled from the oral cavity of rats treated for eight consecutive days, compared to untreated control rats. Treatment with nystatin gave similar results. Histologically, the untreated control animals showed numerous hyphae on the epithelium of the dorsal surface of the tongue. In contrast no hyphal colonization of the epithelium was seen in carvacrol-treated animals, while in rats treated with eugenol, only a few focalized zones of the dorsal surface of the tongue were occupied by hyphae. In the nystatin treated group, hyphae were found in the folds of the tongue mucosa. Thus, the histological data were confirmed by the microbiological tests for carvacrol and eugenol, but not for the nystatin-treated group. Therefore, carvacrol and eugenol could be considered as strong antifungal agents and could be proposed as therapeutic agents for oral candidiasis.

Key Words: Carvacrol, eugenol, experimental oral candidiasis, antifungal activity, essential oils.
\end{abstract}

Oropharyngeal candidiasis is the most common opportunistic infection associated with oral injuries [13]. The expression of Candida albicans virulence in the oral cavity is strongly correlated with impairment of the immune system, particularly in patients with human immunodeficiency virus infection [4]. In addition, several conditions, such as hyposalivation, diabetes mellitus and prolonged antibiotic and corticoid therapy can predispose to oral candidiasis [5]. Specific features of this fungus that contribute to the development of oral candidiasis include its ability to adhere and to colonize the oral mucosa [6] and to form germ tubes [7].

Received on 10 December 2003; revised 27 May 2004.

Address for correspondence: Dr. Remmal Adnane. E-mail: adnaneremmal@hotmail.com. Phone: 212615323 98. Fax: 212 55732981.

The Brazilian Journal of Infectious Diseases 2004;8(3):217-226 (C) 2004 by The Brazilian Journal of Infectious Diseases and Contexto Publishing. All rights reserved.
A number of antifungal agents are available for the treatment of candidal infection [8]. The major agents belong either to the polyenes, such as amphotericin B and nystatin, or to the azoles, such as itraconazole and fluconazole $[9,10]$. Recent studies have indicated $C$. albicans resistance to azoles [11] or hepatotoxicity and nephrotoxicity linked to polyene use, particularly amphotericin B [12]. In order to avoid all these drawbacks, a search for new and effective products is needed to treat this fungal infection.

The antifungal effect of essential oils (EO) of many aromatic plants has been described in several studies $[13,14]$. Specific anticandidal activity is also well established $[15,16]$. The phenolic major components of EO have been suggested to have a potent antifungal activity [17]. Carvacrol, the major component of oregano and thyme oils, and eugenol, the main component of clove oil, were used in this study. We used an experimental model of immunosuppressed rats 
to evaluate the efficacy of these two substances for the treatment of oral candidiasis $[18,19]$. To our knowledge, this is the first time that the anticandidal activity of carvacrol and eugenol was investigated in an oral candidiasis model.

\section{Material and Methods}

\section{Antifungal agents}

Carvacrol and eugenol were purchased from Sigma and Nystatin from Bristol-Myers Squibb.

\section{Animals}

Male Wistar rats ( $\mathrm{n}=41)$ (8 weeks; approximately $200 \mathrm{~g}$ ) were used in this study. They were randomized into groups of three or four animals, housed in large cages. The photoperiods were adjusted to 12 hours of light and 12 hours of darkness, daily. The environmental temperature was constantly maintained at $21^{\circ} \mathrm{C}$. The rats were given ad libitum access to food and water. During the experiment, food composition was complete and equilibrated, free from antifungal agents. The research complied with European legislation and company policy on the care and use of animals and with related codes of practice.

\section{Organisms and inoculum preparation}

Candida albicans (1E111PV515) was isolated from the vaginal secretions of a patient suffering from acute vaginitis. A single colony from Sabouraud glucose agar was grown in yeast extract-peptone glucose medium YPG: (yeast extract, 2\%; bacto peptone, $1 \%$; glucose, $2 \%$ ) for 18 hours at $30^{\circ} \mathrm{C}$ in a shaker. The culture was harvested by centrifugation at $2500 \mathrm{~g}$, then cells were washed three times in phosphate buffer saline (PBS) and adjusted to a final concentration of $3 \times 10^{8} \mathrm{CFU} / \mathrm{mL}$ (using a hemocytometer chamber for counting cells). The viability of the inoculum was confirmed by quantitative cultures of serial 10 fold dilutions on Sabouraud dextrose agar plates. The number of viable cells was determined using the drop count method [20].

\section{Oral candidiasis in the rat}

We used a model of oral candidiasis in immunosuppressed rats that was reported by Martinez et al. $[18,19]$. Our experiments were repeated three times. To enhance the infection rate, rats were immunosuppressed with dexamethasone (Corthametasone, vetoquinol) and treated with tetracycline (Laprophan). One week before infection, rats received drinking water with $0.5 \mathrm{mg} / \mathrm{L}$ of dexamethasone with tetracycline $(0.1 \%)$. On the day of infection, dexamethasone was raised to $1 \mathrm{mg} / \mathrm{L}$, while tetracycline was reduced to $0.01 \%$ and maintained throughout the experiment. The rats were orally infected three times at $48 \mathrm{~h}$ intervals (days- $7,-5$ and -3 ) with $0.1 \mathrm{~mL}$ of saline suspension containing $3.10^{8}$ viable cells of $C$. albicans. A schematic diagram of the oral candidiasis model is presented in Graphic 1.

Oral infection was achieved by means of a cotton swab rolled twice over all parts of the mouth. Just before inoculation, the animals were sampled to confirm the absence of $C$. albicans in the oral cavity, and $72 \mathrm{~h}$ after the last inoculation all groups were sampled in the same manner to check for the presence of the fungi and to quantify the number of CFU in the oral cavity before the beginning of the treatment.

Minimal inhibitory concentrations (MIC) determination:

The lowest concentration of carvacrol, eugenol or nystatin that causes complete inhibition of growth of C. albicans in vitro was determined in triplicate in liquid medium by contact of the antifungal agent with yeast cells for $24 \mathrm{~h}$ at $30^{\circ} \mathrm{C}$, according to the method described by Remmal et al. [21,22].

\section{Oral suspension for topical treatment}

Carvacrol and eugenol at the final concentration 13 $\mathrm{mM}(2 \times \mathrm{MIC})$ and $24 \mathrm{mM}(2 \times \mathrm{MIC})$ respectively were dispersed in viscous $0.8 \%$ agar solution as an excipient for oral treatment. An aqueous solution of 
Graphic 1. Schematic diagram of the oral candidiasis model in immunosuppressed rats reported by Martinez et al. [18] with some modifications

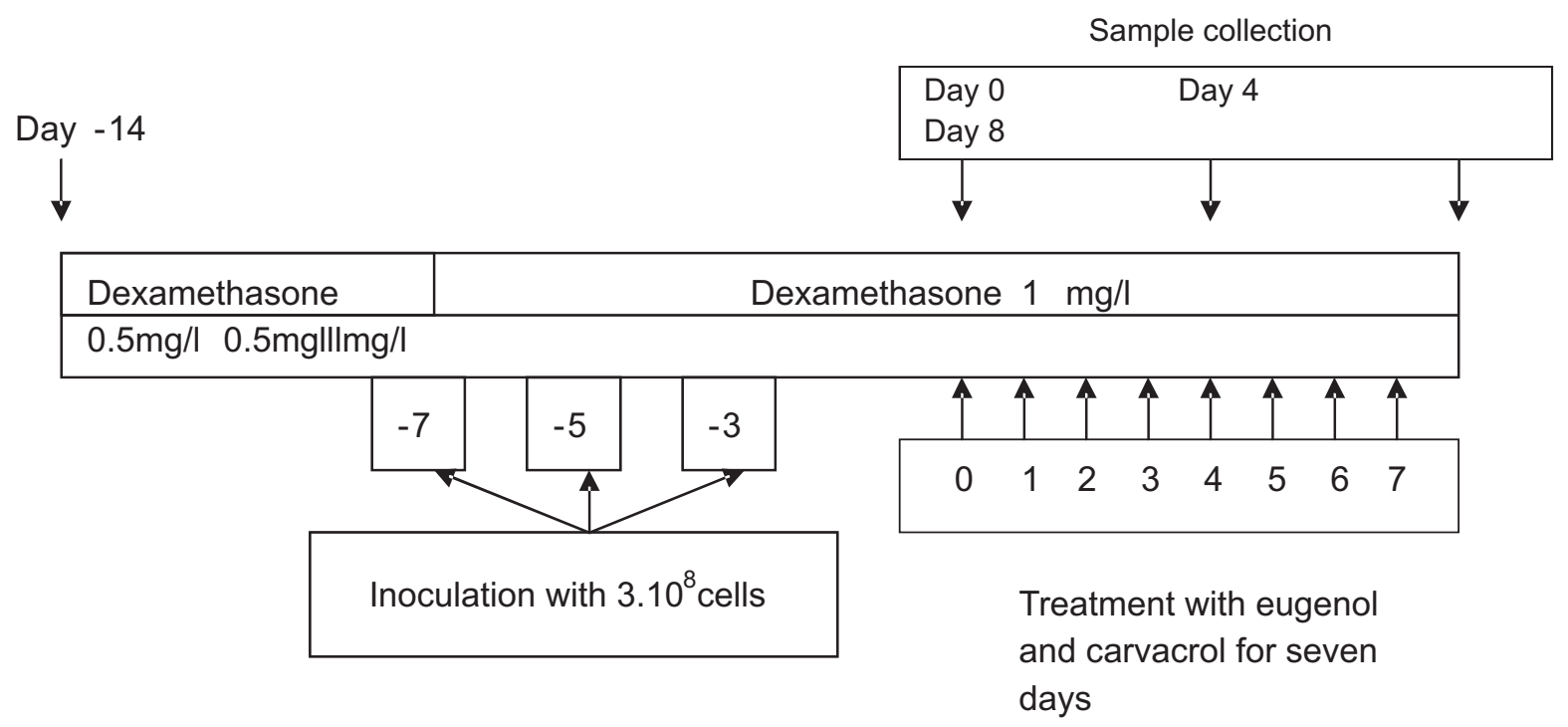

ten fold MIC ( $58 \mu \mathrm{M})$ nystatin was also homogenized in $0.8 \%$ agar.

\section{Antifungal treatment}

Seventy-two hours post infection the animals $(n=41)$ were randomized into six groups. Three of them were treated by topical application on the oral cavity twice a day for eight consecutive days (from day 0 to day 7 ) with $0.5 \mathrm{~mL}$ of carvacrol (13 mM), $0.5 \mathrm{~mL}$ eugenol (24 $\mathrm{mM})$ or $0.5 \mathrm{~mL}$ nystatin suspensions $(58 \mu \mathrm{M})$.

The control, infected and untreated animals received $0.5 \mathrm{~mL}$ sterile saline orally (containing $0.8 \%$ agar) twice a day.

An additional group of animals infected, but not immunosuppressed was added to the experiment to study the impact of dexamethasone/tetracycline treatment on the development of the infection.

Animals immunosuppressed but non infected $(n=6)$ were used as a negative control group.

\section{Quantification of infection level and determination of therapeutic efficacy}

The quantification of the infection was assessed by microbiological and histopathological techniques $[18,19]$.

\section{Microbiology}

Samples were collected at days: 0, 4 and 8 (i.e. 24 hours after the last treatment) by rolling a sterile cotton swab over the oral cavity, which was then suspended in $1 \mathrm{~mL}$ of sterile saline. $25 \mu \mathrm{l}$ samples from this suspension were dropped in duplicate, after serial ten fold dilution on Sabouraud agar plates containing $0.05 \%$ chloramphenicol. All plates were incubated at $30^{\circ} \mathrm{C}$ for $24 \mathrm{~h}$, and the colonies were counted. The number of viable cells was determined using the drop count method [20] to calculate the log of the CFU/mL.

\section{Histopathology}

At day 8, i.e. $24 \mathrm{~h}$ after the administration of the last dose of antifungal agent or saline, the animals were sacrificed with an overdose of ethyl urethane. The tongues were removed, fixed in toto by immersion in Bouin solution for at least $48 \mathrm{~h}$. Tongue sections were embedded in paraffin and $5 \mu \mathrm{m}$ thick serial transverse sections were stained with both hematoxylin-eosin stain and periodic acid Schiff (PAS), to assess the fungal infection. 
Table 1. Microbiological study of therapeutic efficacy of eugenol and carvacrol versus nystatin against oral candidiasis in rats

\begin{tabular}{|c|c|c|c|c|c|c|c|c|}
\hline & \multicolumn{2}{|c|}{ Day 0} & \multicolumn{3}{|c|}{ Day 4} & \multicolumn{3}{|c|}{ Day 8} \\
\hline & $\begin{array}{l}\text { infected } \\
\text { animals } \\
(\%)\end{array}$ & $\begin{array}{c}\log \text { CFU } \\
\text { SD }\end{array}$ & $\begin{array}{c}\text { Positive } \\
\text { culture }(\%)\end{array}$ & $\begin{array}{l}\log \mathrm{CFU} \\
\quad \pm \mathrm{SD}\end{array}$ & $\begin{array}{l}\text { CFU } \\
\text { reduction }\end{array}$ & $\begin{array}{c}\text { Positive } \\
\text { culture }(\%)\end{array}$ & $\begin{array}{l}\log \mathrm{CFU} \pm \\
\quad \pm \mathrm{SD}\end{array}$ & $\begin{array}{c}\text { CFU } \\
\text { reduction } \\
(\%)\end{array}$ \\
\hline Positive control & 7/7 (100) & $3.71 \pm 0.48$ & $7 / 7(100 \%)$ & $3.8 \pm 0.74$ & & $7 / 7(100 \%)$ & $3.71 \pm 0.48$ & \\
\hline Eugenol & 7/7 (100) & $3.62 \pm 0.29$ & $7 / 7(100 \%)$ & $2.97 \pm 0.38 *$ & 95.91 & $2 / 7(28 \%)$ & $3.17 \pm 0.29 *$ & 76.93 \\
\hline Carvacrol & 7/7 (100) & $3.31 \pm 0.46$ & $7 / 7(100 \%)$ & $3.08 \pm 0.46^{*}$ & 91.18 & $3 / 7(42 \%)$ & $2.64 \pm 0.02 *$ & 94.46 \\
\hline Nystatin & $7 / 7$ (100) & $3.64 \pm 0.08$ & $4 / 7(57 \%)$ & $3.07 \pm 0.58 *$ & 96.62 & $1 / 7(14.2 \%)$ & $2.71 *$ & 91.54 \\
\hline $\begin{array}{l}\text { Non Immuno- } \\
\text { suppressed rats }\end{array}$ & $5 / 7$ (71.7) & $3.09 \pm 0.25$ & $2 / 7(28 \%)$ & $3.25 \pm 0.35$ & 92.62 & $1 / 7(14.2 \%)$ & 2.60 & 64.96 \\
\hline
\end{tabular}




\section{Statistical test}

The Student's t-test was used to determine the significance of the observed differences [23].

\section{Results}

In vitro determination of the minimal inhibitory concentrations (MICs) of eugenol and carvacrol against the $C$. albicans strain administrated were made prior to evaluating the therapeutic efficacy of eugenol and carvacrol in this experimental model of oral candidiasis in immunosuppressed rats. Minimal inhibitory concentrations of eugenol and carvacrol were respectively, $12 \mathrm{mM}$ and $6.5 \mathrm{mM}$. The concentrations used for the treatment of rats were $2 \times$ MIC for both components. Nystatin was used as a reference treatment; the MIC was $5.8 \mu \mathrm{M}$; $10 \times$ MIC was utilized to eradicate oral infection.

The efficacy of eugenol and carvacrol was compared microbiologically and histologically to the reference treatment for oral candidiasis.

\section{Microbiological data}

Prior to initiating the study, oral cavity cultures of each rat were performed, and no C. albicans organisms were found.

At day 0, i.e. just before the start of the treatment, all groups of infected animals were sampled. The oral swabs were all positive for the presence of $C$. albicans, with a mean $\log \mathrm{CFU} / \mathrm{swab}$ of $3.69 * \pm 0.49(\mathrm{n}=28$ rats). The group of non immunosuppressed rats was partially infected (5/7), with a mean log CFU/swab of $3.09 \pm 0.25$ (Table 1 ).

Four days after the treatment, oral samples were collected and cultured to quantify the $\mathrm{CFU}$ in the oral cavities of the animals of each group. Animals treated with eugenol and carvacrol remained infected but the mean $\log \mathrm{CFU} / \mathrm{swab}$ decreased significantly, with $\mathrm{p}<0.05$ for eugenol and carvacrol, while in the nystatin group, only $4 / 7$ of rats showed a positive C. albicans culture with a significant decrease in mean $\log \mathrm{CFU} /$ swab. Consequently, the degrees of reduction of CFU versus control were $95.9 \%$ for eugenol, $91.18 \%$ for carvacrol and $96.5 \%$ for nystatin (Table 1 ). In the non immunosuppressed rats, only $2 / 7$ of animals were still infected.

After 8 days of treatment (day 8), C. albicans organisms were detected in the oral cavities of only $2 /$ 7 and 3/7 in eugenol and carvacrol treated groups, respectively. Moreover, a significant percentage reduction of $\mathrm{CFU}$ for the eugenol treated group, compared to the control, was observed (76.9\%). An even larger reduction in the carvacrol treated group (94.5\%) was noted, when compared to the control. Treatment with nystatin was apparently effective to microbiologically eradicate this infection, since only $1 /$ 7 of animals remained infected, with a significant reduction of the $\log \mathrm{CFU} / \mathrm{swab}$.

\section{Histological data}

Infected and untreated control animals showed extensive colonization of the epithelium of the dorsal surface of the tongue by numerous hyphae (Figure 1). In animals treated with carvacrol $(\mathrm{n}=7)$, no evidence of $C$. albicans infection was seen histologically (Figure 2). Furthermore, we noted streptococci at the dorsal surface of the tongue.

Animals treated with eugenol $(\mathrm{n}=7)$ showed only a few focal zones of the tongue surface occupied by hyphae (Figure 3); the number of hyphae was quite small. In these two treated groups, no C. albicans was observed in folds. In comparison, under nystatin treatment, hyphae were found in folds (Figure 4). In this group, the quantity of hyphae in the folds was greater than that found in the tongue surface of eugenoltreated animals.

\section{Discussion}

The antimicrobial and antifungal effect of aromatic plant EO has been described in several studies [1315]. Particularly, the phenolic major components of EO have been suggested to have a potent antimicrobial 
Figure 1. Oral candidiasis in immunosuppressed rats. Transverse sections of rat tongue; untreated control group. PAS staining (x 400).

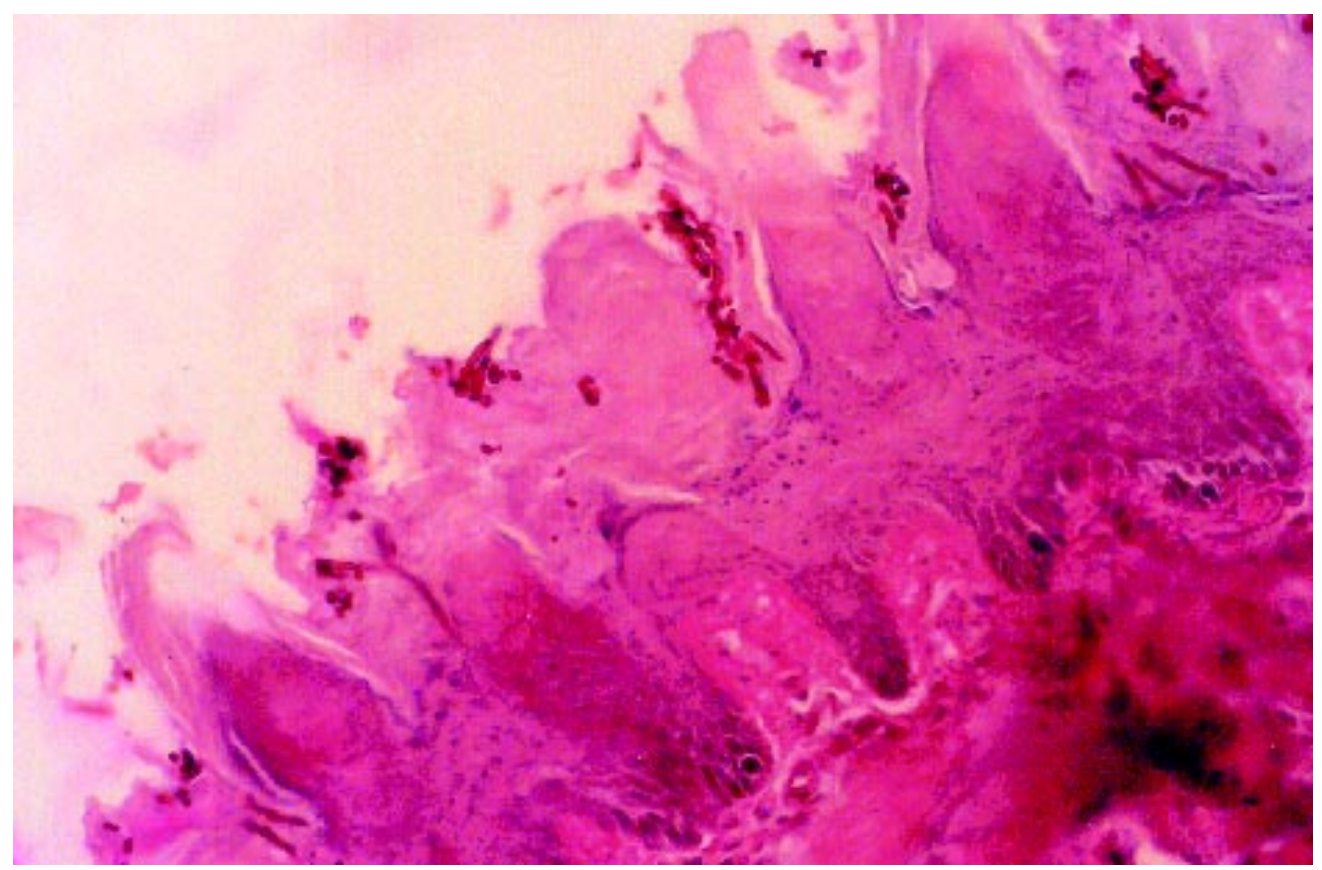

Figure 2. Oral candidiasis in immunosuppressed rats treated with carvacrol $(13 \mathrm{mM})$ twice a day for eight days. Transverse sections of rat tongue; PAS staining x 400. No evidence of Candida albicans infection was seen. Note the presence of streptococci at the surface of the tongue.

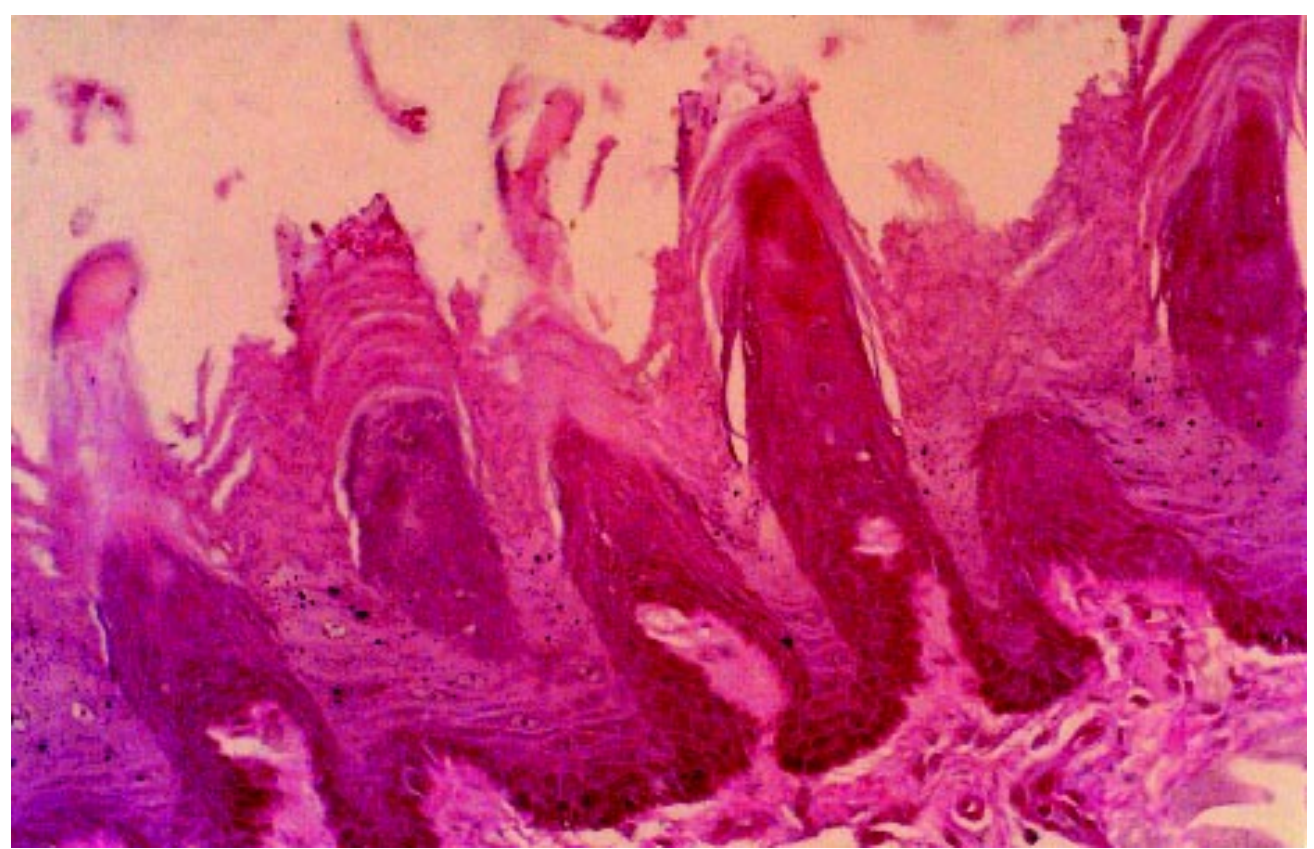


Figure 3. Oral candidiasis in immunosuppressed rats treated with eugenol $(24 \mathrm{mM})$ twice a day for eight days. Transverse sections of rat tongue; PAS staining x 100. Note a few focal zones occupied by hyphae (arrows).

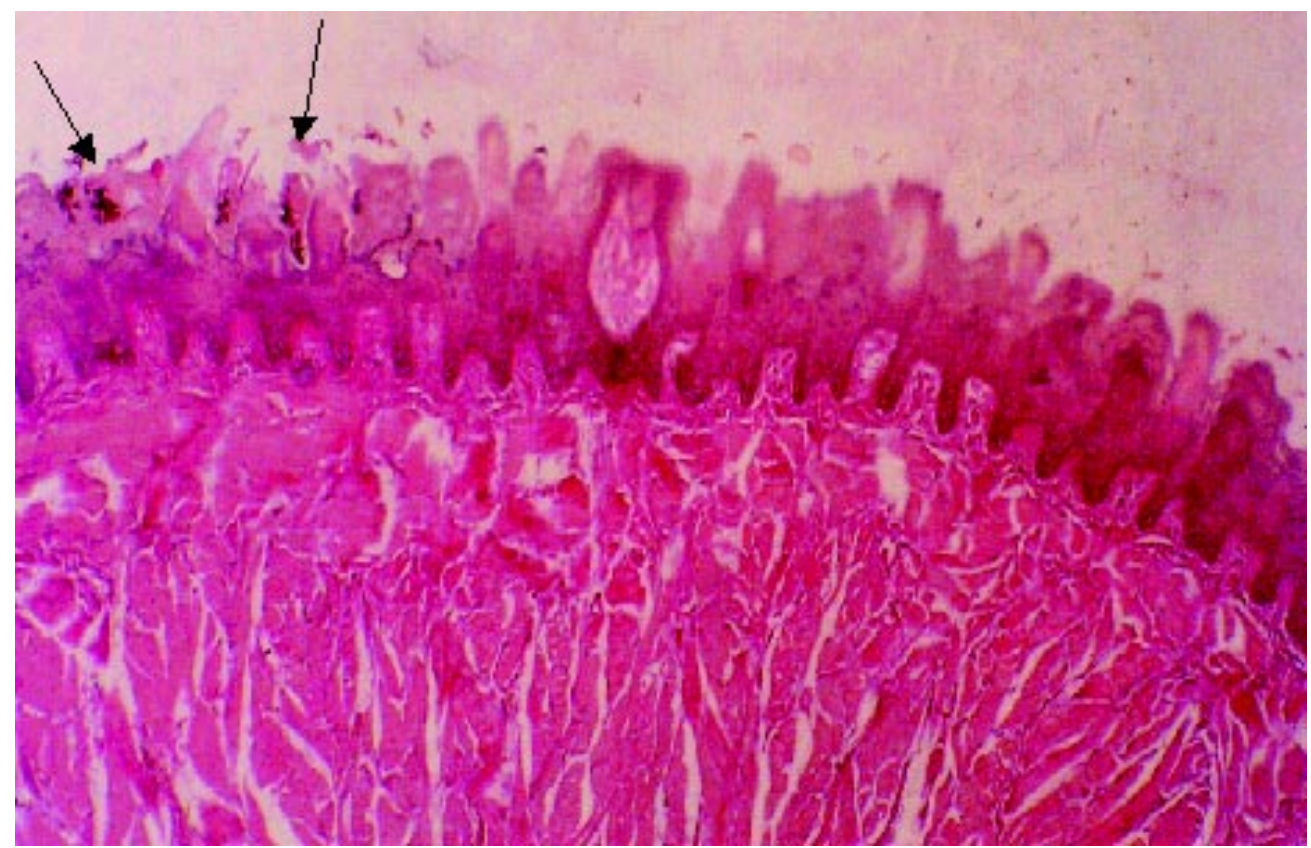

Figure 4. Oral candidiasis in immunosuppressed rats treated with nystatin $(58 \mu \mathrm{M})$ twice a day for eight days. Transverse sections of rat tongue; PAS staining x 100. Note that zones occupied by hyphae are reduced and few hyphae were found in the mucosal folds (arrows).

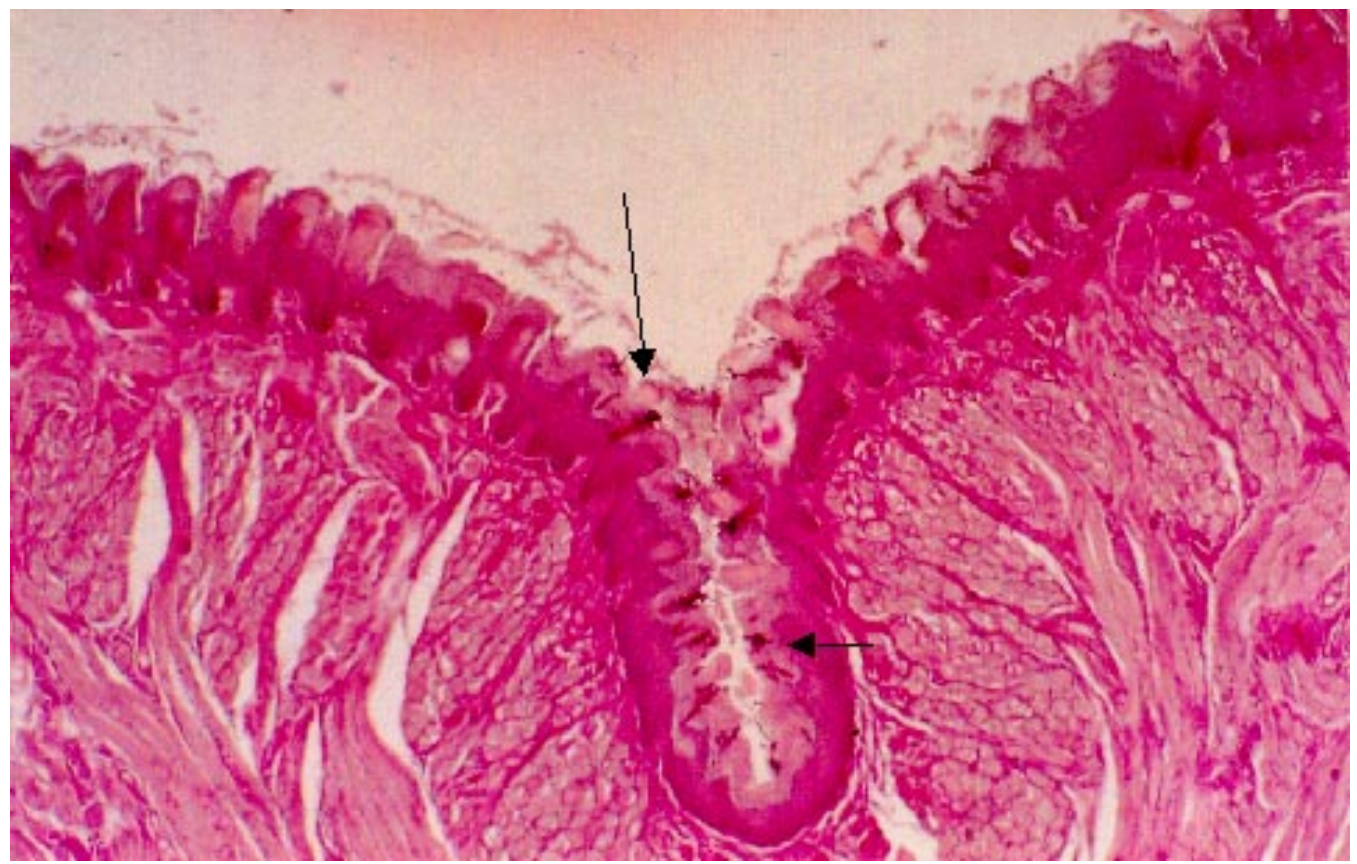


activity in vitro [17,24,25]. Specific anticandidal effects of these major components have been described in vitro $[26,27]$. But up till now, nothing has been reported concerning this effect on oral candidiasis.

The experimental model of oral candidiasis in rats has been shown to be a simple and highly reproducible in vivo method of studying the efficacy of antifungal agents [29].

We have tested the in vivo antifungal activity of two major components of EO, carvacrol and eugenol, on experimental oral candidiasis in immunosuppressed rats; nystatin was used as a positive control treatment. We also assessed the antifungal properties of eugenol and carvacrol against $C$. albicans in vitro. The minimal inhibitory concentration (MIC) of carvacrol and eugenol obtained in vitro were $6.5 \mathrm{mM}$ and 12 $\mathrm{mM}$, respectively, showing that carvacrol is more efficacious than eugenol. These results are similar to those obtained with the same products under the same conditions against bacteria [21-22]. To achieve good adhesion of our products on the oral cavity, a gelatinous suspension of $0.8 \%$ agar was used as an excipient. For topical treatment, we used eugenol and carvacrol at $2 \times$ MIC in vitro, which could be a good choice for the management of this local infection. To validate our experimental conditions, we used a reference treatment group with $10 \mathrm{x}$ MIC of nystatin to eradicate the infection.

The strain of $C$. albicans used in this study was isolated from patients suffering from acute vaginitis and passed several times through rats. It demonstrated high pathogenic properties, since untreated animals showed persistent $C$. albicans infection. However, in the group of non immunosuppressed rats, only 1/7 animals were infected on day 8 , demonstrating the requirement of immunossuppression for a successful model. This finding is in agreement with Martinez et al. [18].

The infected untreated group gave positive Candida cultures throughout the experiment. The mean log CFU/ swab was similar to that obtained by other authors $[9,18]$ with the same inoculum size. Histological observations agreed with microbiological data, as abundant mycelium penetration was observed in the tongue epithelium.
Four days treatment with $2 \times$ MIC of eugenol and carvacrol were insufficient to totally eradicate the oral infection, but they reduced the CFU considerably (95.9\% and $91.2 \%$, respectively). After eight days of treatment with carvacrol and eugenol, only 3/7 and 2/ 7 , respectively, of the treated animals gave positive cultures. The reduction in the number of CFU was considerable, when compared to the control.

The histological study of tongue animals treated with eugenol showed a significant decrease in the number of $C$. albicans by microscopic examination, compared to the control, which parallels the previously described microbiological data. In the group treated with carvacrol, histological data showed that the fungi disappeared completely from the tongue mucosa in all animals, even in the folds. Thus, in this group of animals, the microbiological results agreed with the histological ones. However, we observed the presence of streptococci, which are the habitual resident commensal of oral mucosal surfaces [28]; this apparently took place in the mouth after the end of treatment. There was good efficacy of eugenol and carvacrol in the treatment of $C$. albicans infected rats.

When nystatin was used as a positive control treatment, almost the animals were microbiologically cured on day 8 , however, we found many hyphae on histological examination, in the folds of the tongue of treated animals. In contrast, under eugenol and carvacrol treatment, no hyphae were found in the folds. This may be due to the volatility of these molecules, which allowed their penetration into inaccessible areas, such as folds.

This discrepancy between good microbiological results and the persistence of hyphae, demonstrated histologically, may be explained by the inability of nystatin to penetrate particular areas, such as tongue folds. The presence of $C$. albicans in folds may provoke recurrence.

Eugenol and carvacrol provoke no acute toxicity in rats. The doses used (approximately 160 and $380 \mu \mathrm{g} /$ $\mathrm{Kg}$ ) are far less than the toxic doses demonstrated by Manohar et al. [27].

In conclusion, eugenol and carvacrol are promising drugs for the treatment of oral candidiasis, and therefore 
further studies on their pharmacokinetic and toxicological behaviors are warranted.

\section{Acknowledgements}

The authors are deeply grateful to Mrs. P. Chevallier for her technical assistance in histology, and to DrA. Aboussekhra for his assistance in improving the English of this manuscript.

\section{References}

1. Odds F.C. Candida and candidosis: a review and bibliography. Bailliere Tindale, London, United Kingdom, 1988.

2. Samaranayake L.P., Yaacoub H.B. Classification of oral candidosis, p.124-132.In: L.P. Samaranayake and T. W. MacFarlane (ed.), Oral candidosis. Wright, London, united kingdom, 1990.

3. MacPhail L. M., Greenspan D., Dodd C.L., et al. Association of fungal species with oral candidiasis in HIV infection. J Dent Res 1993;72:35.

4. Lopez-Ribot J., McAtee R., Perea S., et al. Multiple resistant phenotypes of Candida albicans coexist during episodes of oropharyngeal candidiasis in human immunodeficiency virus-infected patients.Antimicr ob. Agents Chemother 1999;43:1621-30.

5. Knight L., Fletcher J. Growth of Candida albicans in saliva: Stimulation by glucose associated with antibiotics, corticosteroids and diabetes mellitus. J Infect Dis 1971;123:371-7.

6. Kennedy M.J. Adhesion and association mechanisms of Candida albicans. Curr Top Med Mycol 1988; 2:73169.

7. Casanova M., Cervera A.M., Gosalbo D., et al. Hemin induces germ tube formation. Infect Immune 1997;65:4360-4.

8. McGinnis M.R., Rinaldi M.G. Antifungal drugs: mechanisms of action, drug resistance, susceptibility testing and assays of activity in biological fluids. In V.Lorian (ed.) Antibiotics in laboratory medicine. The Williams and Willkins Co., Baltimore, Md. 1996. p. 176211.

9. Hata K., Kimura J., Miki H., et al. Efficacy of ER-30346, a novel oral triazole antifungal agent, in experimental models of Aspergillosis, Candidiasis and Cryptococcosis. Antimicrob Agents Chemother 1996;40:2243-7.
10. Ellepolla A.N.B., Samaranayake L.P. Oral candidal infections and antimycotics. Crit. Rev Oral Biol Med 2000;11:172-98.

11. Dupont B.F., Dromer F., Improvisi L. The problem of resistance to azoles in Candida. J Mycol Med 1996;6(Suppl. II):12-9.

12. Kauffman C.A., Carver P. L.. Antifungal agents in the 1990s Current status and future developments. Drugs 1997;53:539-49.

13. Knoblock K., Pauli A., Iberl N., et al. Antibacterial and antifungal properties of essential oil components. J Essent Oil Res 1989;1:119-28.

14. Arras G.., Usai M. Fungitoxic activity of essential oils against four postharvest citrus pathogens: chemical analysis of Thymus capitatus oil and its effect in subatmospheric pressure conditions. J Food Prot 2001;64(7):1025-9.

15. Nenoff P., Haustein U.F., Brandt W. Antifungal activity of the essential oil of Melaleuce alternifolia (tea tree oil) against pathogenic fungi in vitro. Skin Pharmacol 1996;9(6):388-94.

16. Suresh B., Sriram S., Dhanaraj S.A. Anticandidal activity of Santolina chamaecyparissus volatile oil. J Ethnopharmacol 1997;55:151-9.

17. Viollon C., Chaumont J.P. Antifungal properties of essential oils and their main components upon Cryptococcus neoformans. Mycopathologia. 1994;128(3):151-3.

18. Martinez A., Regardera J., Jimenez E., et al. Antifungal Efficacy of GM237354, a Sordarin Derivative, in Experimental Oral Candidiasis in Immunosuppressed Rats. Antimicrob Agents Chemother 2001;45:100813.

19. Martinez A., Ferrer S., Santos I., et al . Antifungal activities of two new Azasordarins, GW471552 and GW471558, in experimental models of Oral and Vulvovaginal Candidiasis in Immunosuppressed Rats. Antimicrob Agents Chemother 2001;45:3304-9.

20. Courvalin P., Drugeon H., Flandrois J.P., Goldstein F. Bactericidie: Aspects théoriques et thérapeutiques in: Caillon J., Drugeon H. Dénombrement des bactéries vivantes Eds Maloine Paris,1991.

21. Remmal A., Bouchikhi T., Tantaoui-Elaraki A., et al Inhibition of antibacterial activity of essential oils by Tween 80 and Ethanol in liquid medium. J Pharm Belg 1993a;48(5):352-6.

22. Remmal A., Tantaoui-Elaraki A., Bouchikhi T., et al. Improved method for the determination of antimicrobial activity of Essential oils in Agar Medium. J Essent oil Res 1993;5:1179-84.

23. Schwartz D. Méthodes statistiques à l'usage des médecins et des biologistes Flammarion Medecine Sciences. Paris, 1984. 
24. Cosentino S., Tuberoso C.I., Pisano B., et al. In vitro antimicrobial activity and chemical composotion of Sardinian Thymus essential oils. Lett Appl Microbiol 1999;29 (2):130-5.

25. Ultee A., Bennik M.H.J., Moezelaar R. The phenolic hydroxyl group of carvacrol is essential for action against the food-borne pathogen Bacillus cereus Appl Environ Microbiol 2002;68(4):1561-8.

26. Ali-Shtayeh M.S., Al-Nuri M.A., Yaghmour R.M., et al. Antimicrobial activity of Micromeria nervosa from the Palestinian area. J Ethnopharmacol 1997;58(3):143-7.

27. Manohar V., Ingram C., Gray J., et al. Antifungal activities of origanum oil against Candida albicans. Mol Cel Biochem 2001;228:111-7.

28. Nair R., Samaranayake L.P. The effect of oral commensal bacteria on candidal adhesion to human buccal epithelial cells. J Med Microbiol 1996;45:179-85.

29. Jones J., Russel C., Young C., et al. Tetracycline and the colonization and infection of the mouths of germ-free and conventionalized rats with Candida albicans. J Antimicrob Chemother 1976;2:247-53. 